\title{
Complications of transoral endonasal-controlled combined endoscopic adenoidectomy
}

\author{
Sami Engin Muz'1, Mahmut Huntürk Atilla² \\ 1 Department of Otolaryngology, Ankara Training and Research Hospital, Ankara, Türkiye \\ 2 Department of Otolaryngology, Yenimahalle Training and Research Hospital, Yıldırım Beyazıt University, Ankara, Türkiye
}

Sami Engin Muz, ORCID: 0000-0002-4255-4939

Mahmut Huntürk Atilla, ORCID: 0000-0001-6400-5888

\begin{abstract}
Objective: The purpose of this study was to observe and evaluate the possible complications of Transoral Endonasal-Controlled Combined Endoscopic Adenoidectomy (TECCA) as a subtype of power-assisted endoscopic adenoidectomy (PAEA) in pediatric patients.

Methods: Sixty-seven children aged between 2 and 12 years who had been diagnosed with adenoid hypertrophy and underwent TECCA from April 2013 to December 2016 as a single procedure (without myringotomy or any kind of tonsil surgery at the same time) were included. Preoperative risk assessment results and intraoperative and post-operative complications were evaluated.
\end{abstract}

Results: Intraoperative and early post-operative bleeding was observed in four $(5.97 \%)$ patients. There was no post-operative complication except for bleeding and no patients were found to have residual adenoid tissue during the six-month post-operative follow-up period.

Conclusion: Endonasal endoscopic control during adenoidectomy helps to identify vascular structures that would normally be at risk of being injured with blind resection. Endoscopic viewing also enables complete removal of intranasal adenoid tissue without significant prolongation in operation time.

Keywords: Adenoidectomy, endoscopy, complications. 


\section{Introduction}

Adenoidectomy is one of the most common pediatric surgical procedures performed by the otolaryngology department. It can be performed with or without tonsillectomy and myringotomy. The most commonly used surgical technique is the removal of adenoid tissue with an adenoid curette. $^{[1,2]}$

In the 1990s, advances in endoscopic sinus surgery popularized the use of power-assisted instruments in adenoidectomy operations with endoscopic assistance. Both transnasal and transoral routes may be used for insertion of the endoscope and microdebrider. Each technique has its advantages, disadvantages and complications, but a transnasally inserted endoscope with transoral microdebrider (Transoral Endonasal-Controlled Combined Adenoidectomy-TECCA) has been reported as the most ergonomic technique for power assisted endoscopic adenoidectomy (PAEA). ${ }^{[3]}$

The aim of the present study was to evaluate the complications of TECCA (a specific subtype of PAEA) in the treatment of children with obstructive adenoid hypertrophy.

\section{Materials and Methods}

The study was approved by the ethics committee of Turgut Özal University Faculty of Medicine in accordance with the declaration of Helsinki (approval number: 99950669/125, date: 21 February 2014). This study was specifically aimed at determining the complications of TECCA in children who underwent treatment between April 2014 and December 2016. A total of 112 pediatric patients had undergone adenoidectomy with or without tonsillectomy/myringotomy at our center during this period. The exclusion of patients who underwent additional procedures resulted in a final total of 67 patients who had undergone TECCA without any additional interventions. The complications evaluated in this study were based on the possible complications of PAEA treatment (Table 1). Informed consent was obtained prospectively from all parents whose children were included in the study.

\section{Table 1. Possible complications of PAEA.}

$>$ Intraoperative and post-operative bleeding

$>$ Readmission for dehydration

$>$ Post-operative fever

$>$ Velopharyngeal insufficiency

$>$ Prolonged recovery

$>$ Post-operative respiratory distress

$>$ Intranasal synechiae

$>$ Nasopharyngeal stenosis

$>$ Eustachian tube stenosis

$>$ Soft palate perforation

$>$ Uvula injury

> Temporomandibular joint dislocation

$>$ Atlantoaxial dislocation

> Head and neck pain

\section{Patients and Preoperative Assessment}

The diagnosis of adenoid hypertrophy was based on patient history (obstruction, sleep disturbance, hyponasality, presence of chronic nasal discharge, hearing problems), otorhinolaryngological examination and fiberoptic endoscopic nasal examination. The degree of obstruction necessitating surgery was defined as the presence of $\geq 50 \%$ posterior choanal obstruction with adenoid tissue in symptomatic patients. Adenoid size and obstruction were assessed via endoscopic examination.

We only included patients in which the adenoidectomy procedure was performed as a single procedure in order to be able to accurately assess complications that were a direct result of TECCA. Patients who underwent other additional or related surgeries (tonsillectomy or other tonsil volume reduction surgeries, myringotomy, ventilation tube insertion) at the same time were excluded from the study.

Detailed history of chronic disease, operations, allergies, bleeding disorders and medications were evaluated prior to surgery, and the American Society of Anesthesiologists Physical Status Classification System (ASA) was used to determine predictors of operational risks.

\section{Surgical technique}

After necessary preparations, orotracheal intubation was 
performed under general anesthesia, a shoulder roll was placed and the head was extended. A Davis-Meyer mouth gag was used to retract the jaw and a mouth gag was fixed to an arm attached to the operation table. Decongestant (Oxymethazoline hydrocloride) soaked cotton packs were placed into the nasal cavity for five minutes to increase space for manipulations and to reduce possible mucosal bleeding. The endoscope (rigid, 0 degree, $18 \mathrm{~cm}$ and 2.7 $\mathrm{mm}$ diameter, Storz, Tuttlingen, Germany) was connected to a high definition camera system and transnasal insertion was performed. The powered microdebrider (Medtronic Xomed, Inc., Jacksonville, FL) was inserted transorally to enable simple manipulation of its tip. The shaver console was set at 3000 revolutions per minute (RPM) in the oscillating mode and the resection was started from the caudal portion of the adenoid tissue (Figure 1). In cases where the adenoid tissue obstructed the choana, thus preventing visualization of the caudal portion of the adenoid tissue, the bulky part of the adenoid tissue was shaved with the microdebrider. The depth of resection was determined by ob-

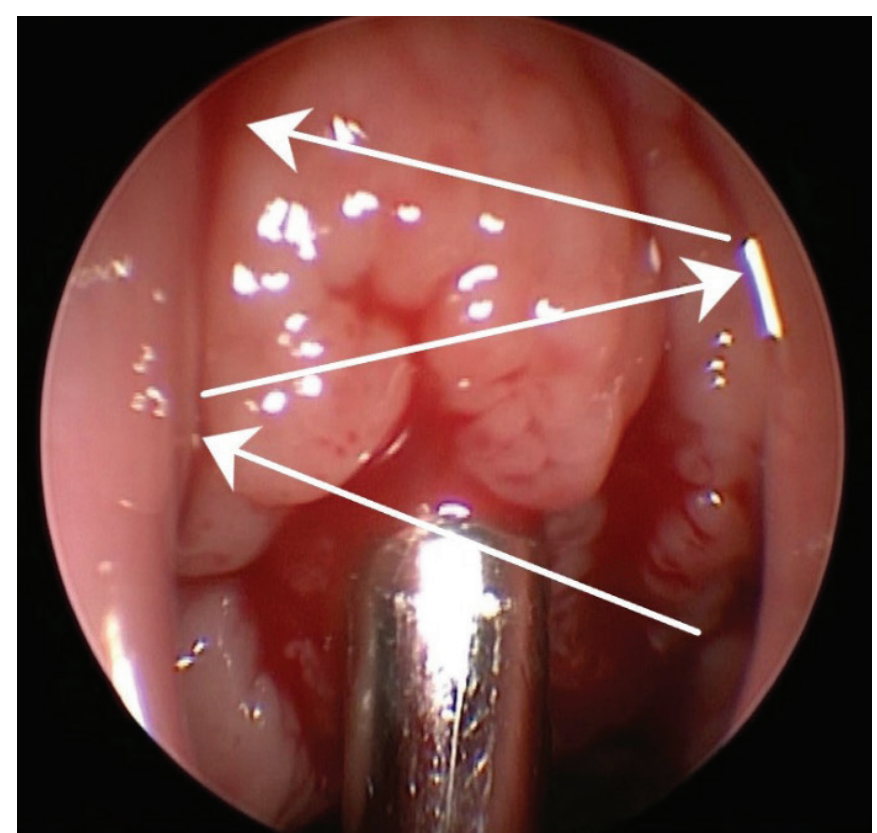

Figure 1. Resection is started from inferior to superior by moving the tip of the microdebrider from one side to another (white arrows). serving the posterior oropharyngeal wall plane and the remaining tissues were checked by palpation of the soft tissue on the vertebral bodies with the tip of the microdebrider. Adenoid tissue proximal to the torus tubarius was resected carefully to avoid damage to the eustachian tube orifice, and any adenoid tissue obstructing the Rosenmüller fossa was also resected in all patients. In the presence of choanal or intranasal adenoids, resection was performed cautiously to avoid damaging the posterior septal branch of the sphenopalatine artery (Figure 2). Due to its regular course above the choana, resection had to be limited to the junction of the choanal mucosa and the adenoid tissue.

Having a clear view of the field is vital and blind resection is contraindicated during all steps of the operation. Continuous suction provided by the microdebrider is essential in removing blood that could limit continuous visual assessment during the operation. After completing all necessary resections, decongestant-soaked sponges were inserted to the nasopharynx for 5 minutes. Suction cautery (Storz Insulated Suction Canula $10 \mathrm{~cm}$ length -

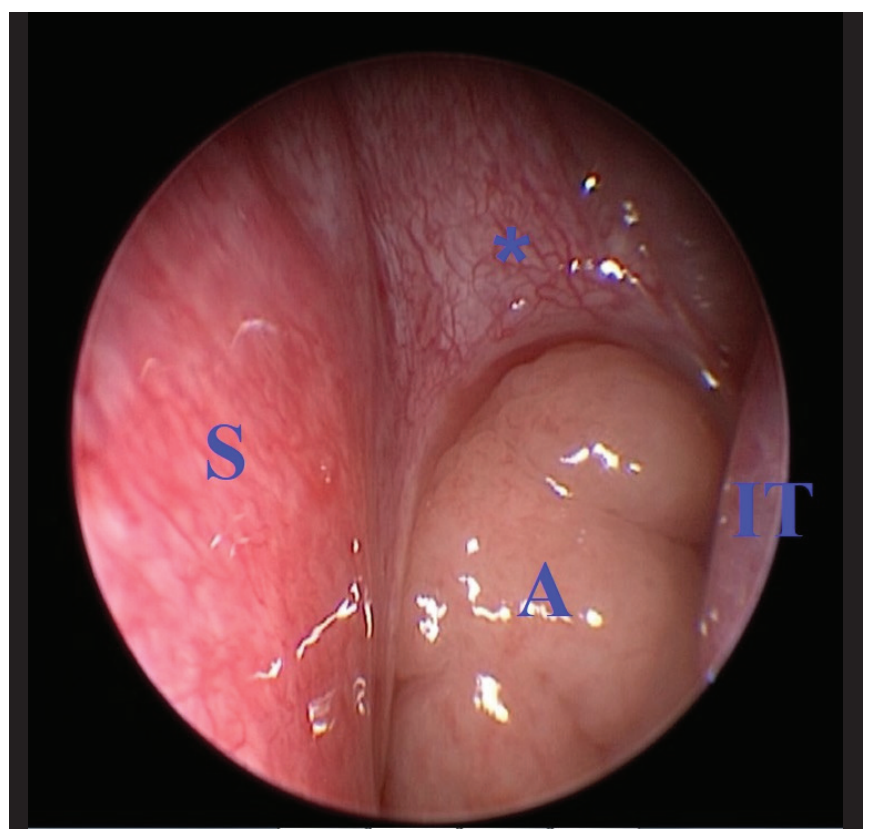

Figure 2. Choanal adenoid tissue extending to the nasal cavity ( $A$ : Adenoid hypertrophy; S: Nasal Septum; IT: Inferior turbinate; * Anterior wall of the sphenoid sinus has rich vascular supply from the posterior septal branches of the sphenopalatine artery). 
$3 \mathrm{~mm}$ diameter, Tuttlingen, Germany) or bipolar cautery (Storz Stammberger Bipolar Suction Forceps 15 or 45 degree, Tuttlingen, Germany) were also utilized to control bleeding and proved to be useful in our experience. Blood loss was calculated as the difference between the amount of irrigation fluid used and the amount of collected fluid in the vacuum container. Blood in cotton packs was often negligible, and was not measured.

\section{Post-operative Follow-Up}

Patients were given antibiotics and nonsteroidal anti-inflammatory drugs for pain during the first 24 hours after surgery. After adenoidectomy, children recover rather rapidly in the postoperative period, and thus discharge on the same day of surgery is acceptable and is often considered to be normal practice. However, in this study, patients were observed in the hospital for bleeding, respiratory distress, pain, fever and other possible complications during the first 24 hours, and were routinely discharged the following morning.

After discharge from the hospital, patients were scheduled for follow-up at the end of the first week and questioned for admission to any hospital due to dehydration, fever, bleeding or pain during the last week. In addition to normal post-operative investigations, velopharyngeal valve movement was also assessed in this visit with a flexible fiberoptic nasopharyngeal endoscope. Two other follow-up studies were scheduled at the third and sixth month after surgery, in which intranasal synechiae, nasopharyngeal stenosis, velopharyngeal insufficiency, eustachian tube stenosis and completeness of resection were checked by endoscopic examination.

\section{Results}

The TECCA procedure was performed as a single procedure in 67 children aged between 2 and 12 years old. Indications for adenoidectomy were nasal obstruction, mouth breathing, snoring and recurrent rhinosinusitis (Table 2). Only one patient (a 10-year-old) had a history of previous curettage adenoidectomy when he was at the age of 4 . All 67 patients were classified as ASA 1 according to preoperative anesthesiological examination.
Table 2. Adenoidectomy indications in children with adenoid hypertrophy

Symptoms Number of children

Nasal obstruction

Mouth Breathing

Snoring

67

Recurrent sinusitis

The patients' adenoid tissue sizes, as determined by endoscopic examination, are given in Table 3 . The majority of patients had $75-90 \%$ obstruction of the posterior choana $(\mathrm{n}=32)$. Total choanal obstruction with extension of adenoid tissue to the nasal cavity was observed in 17 patients (Figure 2).

Table 3. Distribution of the number of children according to the degree of obstruction.

Degree of obstruction Number of children

Between $50 \%-75 \%$

Between $75 \%-90 \% \quad 32$

$>90 \% \quad 13$

The mean resection time was 8 minutes and the average amount of blood loss was about $10 \mathrm{ml}$. Intraoperative bleeding was observed in two patients. One of these was the 10-year-old patient who had a history of previous adenoidectomy. Bipolar cautery was used to control the bleeding but it was not effective. Hemostasis was achieved with the application of decongestant-soaked cotton packs to the area of bleeding for 5 minutes. The overall blood loss of this patient was calculated as $20 \mathrm{ml}$. The second patient had intraoperative pulsatile arterial bleeding because of deep resection. Bleeding was controlled with bipolar cautery and blood loss was $25 \mathrm{ml}$.

Post-operative bleeding was observed in two patients. There were no intraoperative problems in patients with bleeding. Bleeding had started at post-operative 2 hours 
and 3 hours in these patients. Both of them were classified as ASA 1 in preoperative assessment and had no identified risk factor for bleeding. Bipolar cautery was used to obtain hemostasis in both of these children. No other post-operative complications were recorded in any patients throughout follow-up studies extending to the sixth month.

\section{Discussion}

In recent years, various studies have been conducted to evaluate an endoscope-assisted approach in the treatment of adenoidectomy. This approach has been shown to be superior to conventional curettage, by enabling almost-complete elimination of adenoid mass within a shorter surgical duration. ${ }^{[4-6]}$ Also, previous studies demonstrate that the endoscopic approach decreases the frequency of post-operative complications compared to conventional curettage. ${ }^{[5,7,8]}$ Our study was in support of these conclusions.

Although the use of endoscopy allows good vision and provides removal of adenoid tissue with minimal risk of damage to surrounding structures, the endoscopic approach is not risk-free in terms of complications -even though they are mild at most and are easily managed. ${ }^{[9]}$ Primary and secondary hemorrhages are the major complications for all patients undergoing adenoidectomy. Similarly, in the present study, bleeding complications occurred in only $4(5.97 \%)$ patients, including intraoperative bleeding in 2 patients and early post-operative bleeding in 2 patients. None of the patients developed complications other than bleeding and no residual adenoid tissue was observed during the six months of post-operative follow-up. In a meta-analysis study comparing the endoscopic approach and traditional curettage, it was reported that the complication rate was lower with endoscopic treatment $(8.26 \%)$ compared to traditional curettage (30.18\%). ${ }^{[5]}$ In a recent study utilizing the endoscopic adenoidectomy method, bleeding complications were reported in only $2.02 \%$ of patients. ${ }^{[10]}$ Even though the rate of bleeding seems to be lower than our study, the aforementioned study only assessed post-operative bleeding. However, in our study, we evaluated both intraoperative and post-operative complications.

In studies performed with an endoscopic approach, it was reported that the greatest advantages were reduced operation time and intraoperative blood loss compared to traditional methods. ${ }^{[10-12]}$ Somani et al. ${ }^{[13]}$ reported precisely calculated average intraoperative blood loss as $30 \mathrm{ml}$ (range $24-42 \mathrm{ml}$ ) in 44 children in which they had performed different techniques of PAEA treatment. These low values are evidently associated with the fact that endoscopic assistance and continuous blood suction facilitate visual assessment and accurate identification of the vascular plane in the nasopharynx. The latter feature enables prompt evaluation of the source of bleeding, while the prior feature reduces treatment time and blood loss. In relation, the duration of treatment also seems to be positively influenced by these advantages, as Rodriguez et al. ${ }^{[14]}$ have reported that power-assisted partial adenoidectomy (PAPA) resulted in a $59 \%$ reduction in total operating time compared with curettage. Resection time was significantly shorter and time required for hemostasis was also reduced with PAPA. In the present study, average resection time was 8 minutes and intraoperative blood loss was minimal (14 $\mathrm{ml})$. In this regard, our study was compatible with the literature in terms of operation time and intraoperative blood loss.

In general, studies have reported that there are fewer complications with the endoscopic approach; however, studies focused on efficacy are not so unanimous, as several reports have indicated no difference in efficacy between the two methods, while those suggesting that conventional adenoidectomy is better than endoscopic assisted adenoidectomy in terms of blood loss and complications also exist. ${ }^{[15,16]}$ Therefore, it is apparent to us that there is still some virtue in suggesting randomized controlled studies with various treatment options to determine whether the different approaches to endoscopic treatment have varying degrees of success both in terms of treatment outcome and complications.

It should be noted as a limitation of our study that there was no control group in which the endoscopic TECCA approach was compared with other methods. The relatively low number of patients may also be seen as a limitation. However, these were associated with the design of the 
study, as we aimed to evaluate the frequency of complications in TECCA application without any additional/related interventions.

\section{Conclusion}

The TECCA method was found to be a reasonable and safe technique for adenoidectomy in terms of complications and treatment outcome with a follow-up duration of six months. In addition, our experience shows that this method can remove tissue quickly and precisely with minimal blood loss.

Acknowledgments: The authors would like to thank all the children and their families who participated in this study.

Ethics Committee Approval: The study protocol

\section{References}

1. Di Rienzo Businco L, Angelone AM, Mattei A, Ventura L, Lauriello M. Paediatric adenoidectomy: endoscopic coblation technique compared to cold curettage. Acta Otorhinolaryngol Ital 2012;32:124-9.

2. Regmi D, Mathur NN, Bhattarai M. Rigid endoscopic evaluation of conventional curettage adenoidectomy. J Laryngol Otol 2011;125:53-8.

3. Pagella F, Pusateri A, Canzi P, et al. The evolution of the adenoidectomy: analysis of different power-assisted techniques. Int J Immunopathol Pharmacol 2011;24:55-9.

4. Öztürk Ö, Polat Ş. Comparison of transoral power-assisted endoscopic adenoidectomy to curettage adenoidectomy. Adv Ther 2012;29:708-21.

5. Yang L, Shan Y, Wang S, Cai C, Zhang H. Endoscopic assisted adenoidectomy versus conventional curettage adenoidectomy: a meta-analysis of randomized controlled trials. Springerplus 2016;5:426.

6. Havas T, Lowinger D. Obstructive adenoid tissue: an indication for powered-shaver adenoidectomy. Arch Otolaryngol Head Neck Surg 2002;128:789-91.

7. Schupper AJ, Nation J, Pransky S. Adenoidectomy in Children: What Is the Evidence and What Is its Role? Curr Otorhinolaryngol Rep 2018;6:64-73.

8. Pagella F, Pusateri A, Canzi P, et al. The evolution of the adenoidectomy: analysis of different power-assisted techniques. Int J Immunopathol Pharmacol 2011;24:55-9. was approved by the Turgut Özal University Medicine of Faculty Clinical Research Ethics Committee (No: 99950669/135, Date: 21.02.2014).

Informed Consent: Written informed consent was obtained from the parents of each child.

Author Contributions: Designing the study - S.E.M.; Collecting the data - S.E.M., M.H.A.; Analysing the data - S.E.M., M.H.A.; Writing the manuscript - M.H.A.; Con-firming the accuracy of the data and the analyses S.E.M., M.H.A.

Conflict of Interest: The authors have no conflicts of interest to declare.

Financial Disclosure: The authors declare that this study has received no financial support.

9. Costantini F, Salamanca F, Amaina T, Zibordi F. Videoendoscopic adenoidectomy with microdebrider. Acta Otorhinolaryngol Ital 2008;28:269.

10. Saibene AM, Rosso C, Pipolo C, et al. Endoscopic adenoidectomy: a systematic analysis of outcomes and complications in 1006 patients. Acta Otorhinolaryngol Ital 2020;40:79-86.

11. Anand V, Sarin V, Singh B. Changing Trends in Adenoidectomy. Indian J Otolaryngol Head Neck Surg 2014;66:375-80.

12. Stanislaw P Jr, Koltai PJ, Feustel PJ. Comparison of power-assisted adenoidectomy vs adenoid curette adenoidectomy. Arch Otolaryngol Head Neck Surg 2000;126:845-9.

13. Somani SS, Naik CS, Bangad SV. Endoscopic adenoidectomy with microdebrider. Indian J Otolaryngol Head Neck Surg 2010;62:427-31.

14. Rodriguez K, Murray N, Guarisco JL. Power-assisted partial adenoidectomy. Laryngoscope 2002;112:26-8.

15. Elnashar I, El-Anwar MW, Basha WM, AlShawadfy M. Objective assessment of endoscopy assisted adenoidectomy. Int J Pediatr Otorhinolaryngol 2014;78:1239-42.

16. Songu M, Altay C, Adibelli ZH, Adibelli H. Endoscopic-assisted versus curettage adenoidectomy: a prospective, randomized, double-blind study with objective outcome measures. Laryngoscope 2010;120:1895-9.

This is an open access article distributed under the terms of the Creative Commons Attribution-NonCommercial-NoDerivs 3.0 Unported (CC BY- NC-ND3.0) Licence (http://creativecommons.org/licenses/by-nc-nd/3.0/) which permits unrestricted noncommercial use, distribution, and reproduction in any medium, provided the original work is properly cited.

Please cite this article as: Muz S.E., Atilla M.H. Complications of transoral endonasal-controlled combined endoscopic adenoidectomy. ENT Updates 2020;10(3): 390-395. 